\title{
Estado actual de la investigación en Responsabilidad Social Corporativa a nivel organizativo: consensos y desafíos futuros
}

\author{
Antonia Rodríguez Martínez \\ José Moyano Fuentes \\ Juan José Jiménez Delgado
}

\section{RESUMEN}

Este trabajo pretende mostrar el estado actual de la investigación sobre Responsabilidad Social Corporativa (RSC) a nivel organizativo publicada en las revistas de más alto impacto según la Academic Journal Quality Guide, en el ámbito del Management durante el período 2000-2014. En concreto, aporta una clasificación que evidencia los aspectos donde hay acuerdo entre los investigadores y facilita la labor a cualquier lector que desee aproximarse a la situación actual de la investigación sobre este tema de estudio. Asimismo, identifica los retos a los que se enfrentará la investigación en un futuro, tras señalar las lagunas, inconsistencias y oportunidades encontradas en la revisión de la literatura.

PALABRAS CLAVE: Responsabilidad social, ética empresarial, modelos de negocio, responsabilidad pública, entorno social, gestión estratégica, impacto de la RSC.

CLAVES ECONLIT: A130, G340, L200, M140.

Cómo citar este artículo: RODRÍGUEZ, A., MOYANO, J. \& JIMÉNEZ, J.J. (2015): "Estado actual de la investigación en Responsabilidad Social Corporativa a nivel organizativo: consensos y desafíos futuros", CIRIEC-España, Revista de Economía Pública, Social y Cooperativa, 85, 143-179.

Correspondencia: Antonia Rodríguez Martínez, Personal Técnico Contratado, armartin@ujaen.es; José Moyano Fuentes, Catedrático de Universidad, jmoyano@ujaen.es, y Juan José Jiménez Delgado, Profesor Titular de Universidad, juanjo@ujaen.es. Universidad de Jaén. 


\section{État actuel de la recherche en matière de responsabilité sociale des entreprises au niveau organisationnel : Consensus et défis futurs}

RÉSUMÉ : Cet article vise à montrer l'état actuel des recherches sur la responsabilité sociale des entreprises (RSE) au niveau organisationnel, publiées dans les revues ayant un impact majeur, d'après la revue Academic Journal of Quality Guide, dans le domaine du Management pour la période 2000-2014. Plus précisément, nous proposons une nouvelle classification qui met en relief les aspects sur lesquels les chercheurs sont d'accord, facilitant ainsi la tâche du lecteur qui souhaiterait examiner la situation actuelle de la recherche sur ce sujet. De même, nous identifions les défis auxquels les recherches devront se confronter à l'avenir, en signalant les lacunes, les incohérences et les opportunités détectées au cours de l'examen de la littérature disponible sur ce thème.

MOTS CLÉ : Responsabilité sociale, éthique d'entreprise, modèles d'affaires, responsabilité publique, milieu social, gestion stratégique, impact de la RSE.

\section{The current state of research into Corporate Social Responsibility at the organisational level: areas of consensus and future challenges}

ABSTRACT: This paper aims to show the current state of research on Corporate Social Responsibility (CSR) at the organizational level published in the journals with the highest impact according to the Academic Journal Quality Guide for the Management field over the 2000-2014 period. Specifically, it provides a new classification of the literature that shows the areas where there is agreement among researchers and facilitates the work of any reader who wishes to approach the current situation of research on this topic. It also identifies the challenges that research will face in the future, noting the gaps, inconsistencies and opportunities found in the literature review.

KEY WORDS: Social responsibility, business ethics, business models, public responsibility, social setting, strategic management, CSR impact. 


\section{1.- Introducción}

En la actualidad, la Organización de las Naciones Unidas promueve iniciativas internacionales en materia de Responsabilidad Social Corporativa con el objetivo de fomentar actitudes responsables por parte de la empresa y promover así su implicación en las posibles soluciones a los principales retos mundiales. Por otro lado, ya sea por una verdadera asunción de responsabilidades o por razones estratégicas, cada vez son más las voces que defienden que con la Responsabilidad Social Empresarial ha surgido una nueva forma de hacer negocios que puede contribuir a dar una respuesta más satisfactoria a la aspiración de construir un sistema económico y social más inclusivo y sostenible.

En este trabajo se realiza una revisión de la literatura relativa a los aspectos que se han investigado sobre Responsabilidad Social Corporativa (RSC) a nivel organizativo. La amplitud de este objetivo genérico, derivada del interés suscitado entre los investigadores del ámbito de la gestión, motivó que se decidiera delimitar el período de análisis y acotar las revistas objeto de evaluación. En cuanto al período, y de acuerdo con Carroll (2008), la Responsabilidad Social Corporativa comienza a concebirse como un sistema de gestión a partir del año 2000. En cuanto a las publicaciones objeto de evaluación, y asumiendo la hipótesis de que la relevancia de los resultados publicados está relacionada con la calidad de la publicación donde aparecen, se consideraron las revistas de mayor impacto a nivel mundial en el ámbito del management. Estos dos criterios suponen que este trabajo no haya contemplado las publicaciones realizadas antes del año 2000 y las aparecidas en revistas de menor impacto.

Por otro lado, en la revisión de la literatura efectuada se pretende añadir valor, aportando una clasificación centrada en qué aspectos se han estudiado en Responsabilidad Social Corporativa en los últimos años, a qué consensos se ha llegado y qué trabajo queda por realizar por parte de la comunidad científica, tras un estudio y análisis de las lagunas, inconsistencias y oportunidades encontradas en el marco de los trabajos revisados.

Aunque existen otras revisiones de la literatura en artículos ya publicados, este trabajo se diferencia de ellas. Así, por ejemplo, se diferencia del trabajo de Aguinis y Glavas (2012) que utiliza un enfoque basado en la teoría institucional al igual que lo hacen Hoffman (1999), Bansal y Clelland (2004). Por otra parte, los primeros trabajos en el ámbito de la Responsabilidad Social Corporativa versaban sobre preocupaciones ambientales y éticas. Y el trabajo de Barlett (2003) revisa críticamente la literatura relacionada con la gestión de la ética en las organizaciones, o el trabajo de Trevino et al. (2006) en el que se trata la importancia de un comportamiento ético en las organizaciones a nivel individual, de grupo y organizacional. 
Este artículo agrupa la literatura sobre Responsabilidad Social Corporativa a nivel de empresa descartando aquellos trabajos que hacen referencia a otros niveles de análisis, y aporta nuevas líneas de investigación respecto al concepto de Responsabilidad Social Corporativa y a la medición y resultados de aplicar la Responsabilidad Social Corporativa en el ámbito empresarial. En la clasificación realizada se mostrarán además a nivel práctico actuaciones de Responsabilidad Social Corporativa en el núcleo de la empresa, con ejemplos ya estudiados de cómo la aplicación de Responsabilidad Social conlleva beneficios en los diferentes ámbitos de la organización.

Para llevar a cabo este trabajo el examen y la clasificación de los artículos se realizará en base al criterio de elegir sólo aquéllos en los que Responsabilidad Social constituye su contribución principal, descartando aquellos que tratan aspectos implícitos relacionados.

El desarrollo de este trabajo se ha estructurado del siguiente modo: En primer lugar se describe la metodología empleada para lograr los objetivos mencionados. Posteriormente, tras una revisión y análisis de la literatura, se presentan los artículos que han realizado previamente una revisión de la literatura sobre Responsabilidad Social Corporativa subrayando las diferencias con lo realizado en este trabajo. Seguidamente, se aporta la propuesta de clasificación adoptada para agrupar los trabajos examinados, identificando las líneas de investigación desarrolladas, así como los consensos y divergencias entre los autores. Finalmente, se presentan las principales conclusiones, incidiendo en las limitaciones y retos por acometer.

\section{2.- Metodología}

Para llevar a cabo este trabajo se ha realizado una revisión de los artículos publicados en revistas de prestigio sobre aspectos, actuaciones y resultados relacionados con Responsabilidad Social Corporativa a nivel organizativo. Para ello, se han seleccionado aquellos artículos donde la RSC constituye el núcleo central del mismo y en los que el nivel de análisis empleado es la organización. En este sentido, se han descartado aquéllos que trataban aspectos implícitos o complementarios a la RSC o que utilizaban un nivel de análisis individual o institucional. Esta metodología se ha empleado con éxito en otros trabajos publicados, como en el trabajo de Aguinis y Glavas (2012).

Para identificar las revistas objetivo donde realizar las búsquedas se ha utilizado la Academic Journal Quality Guide utilizando las revistas clasificadas en los rangos 4*, 4 y 3 del ámbito "General Management" de esta guía, garantizando la calidad de las revistas y publicaciones a manejar para realizar la revisión de la literatura. 
Para localizar los artículos se han realizado búsquedas en las principales bases de datos bibliográficas, como ABI Inform Global, SciverseHub (Scopus+ScienceDirect+Scirus), ICYT (Ciencia y Tecnología), ISI Journal Citation Report, Scopus y WebWOK (Web of Knowledge) para el período 20002014. Sin embargo, la búsqueda final se decidió realizar a través de Scopus debido a lo operativo del sistema de búsqueda avanzada y a la eficiencia y efectividad en la delimitación de los campos de búsqueda, siendo idénticos los resultados obtenidos utilizando otras bases de datos.

Las palabras o términos clave que se han empleado son aquéllos que, frecuentemente, se utilizan en la literatura para describir la Responsabilidad Social Corporativa, teniendo en cuenta trabajos como los de Aguinis y Glavas (2012), Crane et al. (2014), McWilliams y Siegel (2001) o Surroca et al. (2013). Para llegar a delimitar las palabras clave primero se realizaron varias búsquedas con términos significativos de manera individual, posteriormente se realizaron búsquedas combinadas de dos o más términos. Con los resultados obtenidos se analizaron los artículos, se observaron las palabras o términos clave y estos llevaron a confeccionar una serie de términos como los más destacables y nombrados en la bibliografía científica relacionados con Responsabilidad Social Corporativa.

De este modo, las palabras clave que han sido consideradas son: "Social Responsibility (teniendo en cuenta que este término engloba Corporate Social Responsibility); Corporate Responsibility; CSR; Corporate Social Performance; CSP; Social Responsiveness; Public Responsibility; Business Ethics; Irresponsibility; Social Responsible Investing; Social Commerce; Corporate Ethics; Corporate Social Involvement; Stakeholder theory; Ethical behavior; Business codes". Estas palabras se introdujeron en las búsquedas por título, resumen y palabras clave.

En base a estas acotaciones las búsquedas realizadas en las bases de datos produjeron centenares de artículos, examinándose cada uno de ellos, con el fin de garantizar que su contribución estaba relacionada directamente con Responsabilidad Social Corporativa. Teniendo en cuenta los criterios y objetivos de esta investigación, primeramente se revisaron los abstracts; una vez que se identificó que su contribución estaba relacionada con el objetivo de este trabajo se analizó en profundidad cada artículo, observando las conexiones y divergencias con otras investigaciones.

Finalmente, se obtuvieron un total de 184 artículos, de los cuales, tras su revisión en profundidad y depuración se eliminaron 63 por no cumplir con los criterios definidos y por utilizar un nivel de análisis distinto al organizativo. Un resumen de la metodología empleada en la investigación se puede ver reflejado en la siguiente tabla: 


\section{Tabla 1. Resumen de la metodología empleada}

\begin{tabular}{|l|l|}
\hline Unidad de análisis & $\begin{array}{l}\text { Artículos relevantes sobre RSC publicados en el ámbito General Management en los } \\
\text { rangos } 4^{*}, 4 \text { y } 3, \text { según la Guía ABI The Association of Business Schools (2010). }\end{array}$ \\
\hline Naturaleza del análisis & Cualitativa \\
\hline Base de Datos & Scopus \\
\hline Período de análisis & $2000-2014$ \\
\hline Palabras clave en búsquedas & $\begin{array}{l}\text { Social Responsibility; Corporate Responsibility; CSR; Corporate Social Performance; CSP; } \\
\text { Social Responsiveness; Public Responsibility; Business Ethics; Irresponsibility; Social } \\
\text { Responsible Investing; Social Commerce; Corporate Ethics; Corporate Social Involvement; } \\
\text { Stakeholder theory; Ethical behavior; Business codes. }\end{array}$ \\
\hline Revistas & $\begin{array}{l}\text { Academy of Management Review*, Academy of Management Journal } \\
\text { Science Quarterly*, Journal of Management, Journal of Management Studies, Harvard } \\
\text { Business Review, British Journal of Management, California Management Review, MIT Sloan } \\
\text { Management Review, International Journal of Management Reviews, Academy of } \\
\text { Management Perspectives, Journal of Management Inquiry. }\end{array}$ \\
\hline Selección & Aquellos artículos en los que Responsabilidad Social Corporativa es el núcleo. \\
\hline Total artículos & 121 \\
\hline
\end{tabular}

FUENTE: Elaboración propia.

En la tabla 2 se refleja el número de publicaciones por revista, siendo destacables las revistas Journal of Management Studies, California Magement Review, Journal of Management, Academy of Management Review y Harvard Business Review, por el mayor número de publicaciones en el período estudiado.

\section{Tabla 2. Número de publicaciones analizadas por revista}

\begin{tabular}{|lc|}
\hline Nombre de la Revista & Número de Publicaciones \\
\hline MIT Sloan Management Review & 4 \\
Journal of Management Studies & 21 \\
Journal of Management Inquiry & 4 \\
Journal of Management & 13 \\
International Journal of Management Reviews & 8 \\
Harvard Business Review & 11 \\
California Management Review & 19 \\
British Journal of Management & 11 \\
Administrative Science Quarterly & 2 \\
Academy of Management Review & 13 \\
Academy of Management Perspectives & 8 \\
Academy of Management Journal & 7 \\
TOTAL & 121 \\
\hline
\end{tabular}

FUENTE: Elaboración propia. 
En el siguiente gráfico (gráfico 1) se reflejan el número y evolución de las publicaciones en el período estudiado (2000-2014), y con ello la importancia que ha cobrado la Responsabilidad Social Corporativa a nivel empresarial.

\section{Gráfico 1. Evolución del número de publicaciones (2000- 2014)}

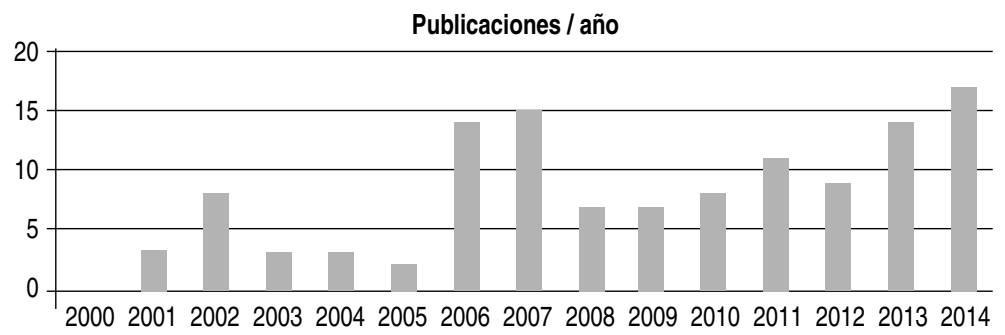

FUENTE: Elaboración propia.

En este gráfico no se observa un patrón claro en el número de artículos publicados en el período analizado. No obstante, sí se detecta que la RSC a nivel organizativo ha sido un tema recurrente y de cierta transcendencia en las revistas de mayor impacto en el ámbito del management, pues desde el año 2006 se han publicado anualmente 6 o más artículos sobre esta temática. Además, en los años 2013 y 2014 se observa un número muy superior de artículos publicados respecto de la media del período y que estaría relacionado con lo indicado por Carroll (2008) y Carroll y Shabana (2010) en cuanto a la mayor preocupación de las empresas sobre el rol que desempeñan en la sociedad.

Para la elaboración de la clasificación de la literatura en base a los criterios previamente establecidos se partió de muchas pequeñas agrupaciones en base a la temática principal del artículo, continuando con una agrupación hacia arriba ("down-top") formando grandes grupos. Hasta llegar a una gran agrupación que englobara los términos a los que se pretendía llegar como pilares de la Responsabilidad Social Corporativa a nivel organizativo. 


\section{3.- Análisis e investigación teórica sobre Responsabilidad Social Corporativa}

En este apartado se analizan revisiones previas de la literatura realizadas sobre Responsabilidad Social Corporativa incidiendo en sus aportaciones fundamentales y mostrando las semejanzas y diferencias respecto de lo realizado en este trabajo.

Aguinis y Glavas (2012) realizan una revisión de la literatura sobre RSC asumiendo la definición dada por Aguinis (2011, p. 855): "acciones organizativas y políticas específicas en el contexto que tengan en cuenta las expectativas de los grupos de interés y la línea de triple cuenta de resultados: rendimiento económico, social y ambiental" incidiendo en que estas acciones y políticas están influenciadas por los actores en todos los niveles de análisis: institucional, organizacional e individual (Aguinis y Glavas, 2012). El presente trabajo difiere del de Aguinis y Glavas (2012) en el nivel de análisis utilizado.

La revisión realizada por Wood (2010) incide en la necesidad de analizar cómo se mide el impacto de la RSC y ver en qué grado es rentable para las empresas, aspecto que también es tenido en cuenta en la clasificación que se aporta en este trabajo.

Por otro lado, existen trabajos de revisión de la literatura que se centran en la influencia de la RSC sobre la maximización de la riqueza y de los resultados empresariales (Laplume, Sonpar y Litz, 2008). Otros autores, sin embargo, indican que toda organización socialmente responsable debe ampliar su enfoque centrándose en una estrategia global y desarrollarla tanto a nivel interno como externo (Peng y Pleggenkuhle-Miles, 2009). Por todo ello, en la revisión de la literatura que se lleva a cabo en este trabajo se tendrá en cuenta tanto la rentabilidad económica que se alcanza a través de la RSC como la necesidad de integrar la RSC en la gestión para alcanzar beneficios a largo plazo.

Gran parte de la investigación sobre RSC refleja su carácter interdisciplinar. Lockett et al. (2006) indican que los autores indagan sobre las diferentes propuestas y conceptos básicos que se plantean e investigan la situación de la RSC dentro de la literatura de gestión, analizando el enfoque y la naturaleza y muestran la importancia de los aspectos teóricos y empíricos centrados en preocupaciones ambientales y éticas. Este estudio se diferencia del de estos autores en que se tratan aspectos teóricos referidos a los tres ámbitos de la RSC (económica, social y medioambiental) a nivel organizacional.

Por otro lado, en este trabajo se plantea atender a un concepto unívoco subrayando la necesidad de normativa legal y efectiva para las empresas y la sociedad en el ámbito de la Responsabilidad Social Corporativa. Scherer y Palazzo $(2007 ; 2011)$ realizan revisiones de la literatura atendiendo principal- 
mente a conceptos politizados de la responsabilidad social. En otro sentido, Lee (2008) propone un enfoque particular en estrecha unión con los objetivos financieros de las organizaciones. Otros autores, como Benn y Bolton (2011) y Secchi (2007), hablan de conceptos y teorías de la responsabilidad social surgidos desde mediados de 1970. Sin embargo, debido a la evolución del significado y al gran número de investigaciones en los últimos años (como refleja el gráfico 1), son necesarios nuevos esfuerzos para comprender los nuevos desarrollos en RSC. En el presente artículo se añade la necesidad de concretar un concepto y reflexionar sobre el camino en la evolución del mismo y las implicaciones para su desarrollo, que abarquen los tres ámbitos de la Responsabilidad Social Corporativa a nivel organizacional.

\section{4.- La investigación en Responsabilidad Social a nivel organizativo}

En este apartado se propone una nueva clasificación de la investigación sobre responsabilidad social a nivel organizativo a partir la revisión de la literatura efectuada y que constituye la principal aportación de este artículo.

De este modo, la literatura revisada puede ser agrupada en tres grandes grupos:

- Un primer grupo que recoge aquellos estudios en los que los investigadores analizan la influencia que tiene el entorno en la RSC a nivel organizativo y de qué manera se puede realizar ese cambio $o$ adaptación.

- Un segundo grupo que engloba aquellos trabajos cuyo tema principal trata sobre cómo las empresas adoptan los procesos previos a la implantación de medidas de RSC en el interior de la empresa o en diferentes sectores.

- Un tercer grupo que recoge los trabajos que analizan el impacto de la RSC a nivel de empresa en tres ámbitos: económico, social y medioambiental.

Cada uno de estos grupos propuestos se subdividen en líneas que contribuyen a la explicación detallada de esta clasificación e identificadas en base a las cuestiones de investigación de los trabajos analizados.

En el esquema reflejado en la figura 1 puede verse la clasificación propuesta: 
Figura 1. Esquema de la clasificación propuesta

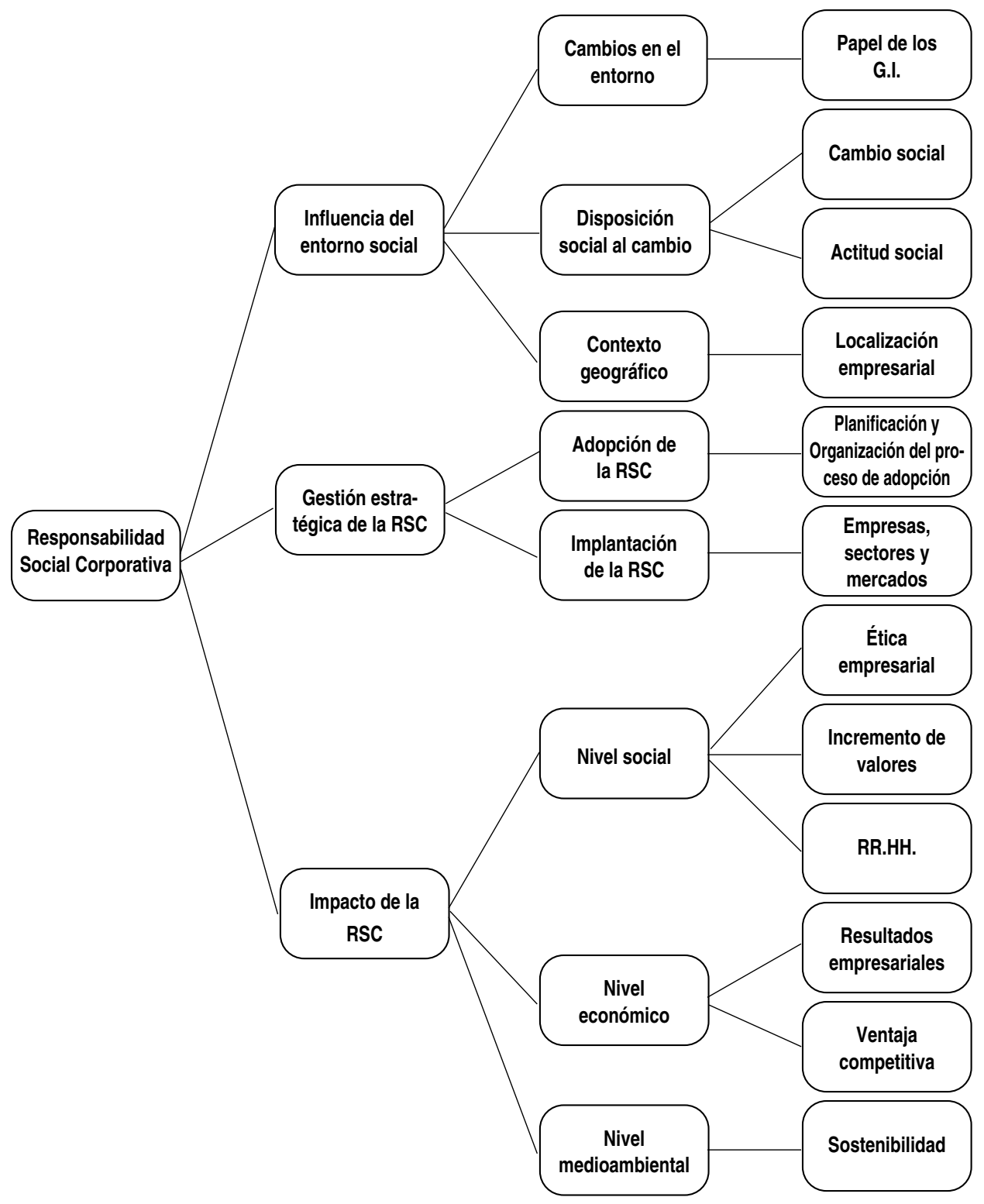

FUENTE: Elaboración propia. 
La siguiente tabla (tabla 3) muestra los trabajos ordenados cronológicamente y agrupados de acuerdo con la clasificación propuesta.

\section{Tabla 3. Agrupación de la literatura}

\begin{tabular}{|c|c|c|c|}
\hline \multirow{4}{*}{$\begin{array}{l}\text { Influencia } \\
\text { del entorno } \\
\text { social }\end{array}$} & $\begin{array}{l}\text { Cambios en } \\
\text { el entorno }\end{array}$ & $\begin{array}{l}\text { Papel de los } \\
\text { grupos de interés }\end{array}$ & $\begin{array}{l}\text { (McWilliams y Siegel, 2001) (Martin, 2002) (Barnett, 2007) (Cheah, Jamali, } \\
\text { Johnson y Sung, 2011) (Vallester, Lindgreen, Maon, Kleyn et al., 2012) (Aguinis, } \\
\text { Forcum, Joo, 2013) (Hillenbrand, Money, Ghobadian, 2013) }\end{array}$ \\
\hline & \multirow[t]{2}{*}{$\begin{array}{l}\text { Disposición } \\
\text { social al } \\
\text { cambio }\end{array}$} & Cambio social & $\begin{array}{l}\text { (Hess, Rogovsky y Dunfee, 2002) (Windsor, 2006) (Carmeli, Gilat y Waldman, } \\
\text { 2007) (Lévy, Eskew, Bernotat y Barner, 2007) (Höllerer, 2013) (Scherer, Palazzo, } \\
\text { Seidl, 2013) }\end{array}$ \\
\hline & & Actitud social & $\begin{array}{l}\text { (Child y Gunther, 2001) (Margolis y Walsh, 2003) (Beck y Ogden, 2007) (Waddock, } \\
\text { 2008) (Rivoli y Waddock, 2011) (Salge, Vera, 2013) (Mena, Waeger, 2014) }\end{array}$ \\
\hline & \begin{tabular}{|l|} 
Contexto \\
geográfico
\end{tabular} & $\begin{array}{l}\text { Localización } \\
\text { empresarial }\end{array}$ & $\begin{array}{l}\text { (Warren y Tweedale, 2002) (Doh y Guay, 2006) (Bailey y Spicer, 2007) (Sharratt, } \\
\text { Brigham y Brigham, 2007) (Matten y Moon, 2008) (Arya y Zhang, 2009) (Surroca, } \\
\text { Tribó, Zahra, 2013) (Bondy, Starkey, 2014) (George, 2014) }\end{array}$ \\
\hline \multirow[t]{2}{*}{$\begin{array}{l}\text { Gestión } \\
\text { estratégica } \\
\text { de la RSC }\end{array}$} & $\begin{array}{l}\text { Adopción de la } \\
\text { RSC }\end{array}$ & $\begin{array}{l}\text { Planificación y } \\
\text { organización } \\
\text { del proceso de } \\
\text { adopción }\end{array}$ & $\begin{array}{l}\text { (Smith, 2003) (Zadek, 2004) (Husted y De Jesus Salazar, 2006) (McWilliams, } \\
\text { Siegel y Wright, 2006) (Porter y Kramer, 2006) (Aguilera, Rupp, Williams y } \\
\text { Ganapathi, 2007) (Berger, Cunningham y Drumwright, 2007) (Carroll y Shabana, } \\
\text { 2010) (Galbreath, 2010) (Karnani, 2011a) (Nonaka y Takeuchi, 2011) (Den Hond, } \\
\text { Rehbein, Bakker, Lankveld, 2014) (Raffaelli, Glynn, 2014) }\end{array}$ \\
\hline & $\begin{array}{l}\text { Implantación } \\
\text { de la RSC }\end{array}$ & $\begin{array}{l}\text { Empresas, } \\
\text { sectores y } \\
\text { mercados }\end{array}$ & $\begin{array}{l}\text { (Brugmann y Prahalad, 2007) (Campbell, 2007) (Mackey, Mackey y Barney, 2007) } \\
\text { (Basu y Palazzo, 2008) (Lindgreen y Swaen, 2010) (Maon, Lindgreen y Swaen, } \\
\text { 2010) (Chatterjee, 2013) (Christensen, Mackey, Whetten, 2014) (Ross, 2014) }\end{array}$ \\
\hline \multirow{6}{*}{$\begin{array}{l}\text { Impacto de } \\
\text { la RSC }\end{array}$} & \multirow[t]{3}{*}{ Nivel social } & Ética empresarial & $\begin{array}{l}\text { (Bos y Willmott, 2001) (Bartlett, 2003) (Jacobs, 2004) (Oosterhout, Heugens y } \\
\text { Kaptein, 2006) (Trevino, Weaver y Reynolds, 2006) (Clegg, Kornberger y Rhodes, } \\
\text { 2007) (Kaptein, 2008) (Barraquier, 2011) (De Cremer, van Dick, Tenbrunsel, } \\
\text { Pillutla y Murnighan, 2011) (Kleyn, Abratt, Chipp y Goldman, 2012) (Chin, } \\
\text { Hambrick, Treviño, 2013) (Lllia, Zyglidopoulos, Romenti, Rodriguez-Canovas, Del } \\
\text { Valle Brena, 2013) (Bazerman, 2014) (Contu, 2014) (Godfrey, Mahoney, 2014) } \\
\text { (Weaver, Reynolds, Brown, 2014) (Welsh, Ordóñez, 2014) }\end{array}$ \\
\hline & & $\begin{array}{l}\text { Incremento de } \\
\text { valores }\end{array}$ & $\begin{array}{l}\text { (Soule, 2002) (Hollender, 2004) (Waldman, Siegel y Javidan, 2006) (Eccles, } \\
\text { Newquist y Schatz, 2007) (Berry y Robinson, 2009) (Devinney, 2009) (Janney y } \\
\text { Gove, 2011) (Minor y Morgan, 2011) (Dowling y Moran, 2012) (Lange y Washburn, } \\
\text { 2012) (Barnett, 2014) (Cording, Harrison, Hoskisson, Jonsen, 2014) (Crane, } \\
\text { Palazzo, Spence, Matten, 2014) }\end{array}$ \\
\hline & & RR.HH. & $\begin{array}{l}\text { (Frenkel y Scott, 2002) (Bhattacharya, Sen y Korschun, 2008) (Muthuri, Matten y } \\
\text { Moon, 2009) (Mirvis, 2012) }\end{array}$ \\
\hline & \multirow[t]{2}{*}{$\begin{array}{l}\text { Nivel } \\
\text { económico }\end{array}$} & $\begin{array}{l}\text { Resultados } \\
\text { empresariales }\end{array}$ & $\begin{array}{l}\text { (Mintzberg, Simons y Basu, 2002) (Porter y Kramer, 2002) (Pearce y Doh, 2005) } \\
\text { (Vogel, 2005) (Deckop, 2006) (Freeman, 2006) (Schuler y Cording, 2006) (Doh, } \\
\text { Howton, Howton y Siegel, 2009) (Peloza y Falkenberg, 2009) (Du, Bhattacharya y } \\
\text { Sen, 2010) (Karnani, 2011b) (Tang, Hull y Rothenberg, 2012) (Chun, Shin, Choi, } \\
\text { Kim, 2013) (Wang y Choi, 2013) (Oikonomou,Brooks, Pavelin, 2014) }\end{array}$ \\
\hline & & $\begin{array}{l}\text { Ventaja } \\
\text { competitiva }\end{array}$ & $\begin{array}{l}\text { (Goold y Campbell, 2002) (Marcus y Anderson, 2006) (Delios, 2010) (McWilliams y } \\
\text { Siegel, 2011) (Orlitzky, 2013) }\end{array}$ \\
\hline & $\begin{array}{l}\text { Nivel medio- } \\
\text { ambiental }\end{array}$ & Sostenibilidad & $\begin{array}{l}\text { (Brammer y Pavelin, 2006) (Kock, Santaló y Diestre, 2012) (Peloza, Loock, Cerruti } \\
\text { y Muyot, 2012) (Delmas, Etzion, Nairn-Birch, 2013) (Flammer, 2013) (Lampikoski, } \\
\text { Westerlund, Rajala, Möller, 2014) }\end{array}$ \\
\hline
\end{tabular}

FUENTE: Elaboración propia. 


\subsection{Influencia del entorno social}

Uno de los tópicos sobre los que ha girado la investigación en RSC a nivel organizativo es el papel del entorno social en la adopción de actitudes y comportamientos de responsabilidad social en las empresas. Dentro de este tópico se han identificado tres líneas de investigación diferenciadas que analizan, por un lado, el papel de los cambios en el entorno, la disposición social para ejecutar cambios en la organización y, por último, cómo influye de diferente manera el entorno social en las actuaciones de RSC a nivel de empresa.

\subsubsection{Cambios en el entorno}

Las actuaciones de RSC indican el camino para conseguir el cambio en todos los niveles de la empresa hacia los grupos de interés. Para ello será necesario un cambio en el pensamiento y los valores de la empresa a todos los niveles, comenzando por los directivos y accionistas, trabajadores y resto de grupos de interés afines a la organización. En este epígrafe se hace referencia al papel del entorno social en la RSC de la empresa.

\subsubsection{El Papel de los Grupos de Interés}

En este apartado se analiza la importancia derivada de incluir en actuaciones empresariales socialmente responsables a otros grupos de interés externos a la propia empresa. Como argumentan McWilliams y Siegel (2001), existe un nivel ideal de RSC que los gerentes pueden determinar a través del análisis coste-beneficio existiendo relación entre responsabilidad social y rendimiento financiero. En esta misma línea, Hillenbrand et al. (2013), indican que los beneficios de la RSC para las organizaciones deben alcanzarse a través de un mayor apoyo y confianza de los grupos de interés.

En el ámbito de la organización aparece un nuevo grupo, los Inversores Socialmente Responsables (SRI), como uno de los grupos de interés que se encuentran en el entorno empresarial, siendo destacables los resultados del estudio de Cheah et al. (2011) que demuestran que los SRI pueden ser impulsores de la RSC en el entorno empresarial. Siendo importante también tratar el mercado, como grupo de interés, Aguinis et al. (2013) lo analizan identificando las relaciones entre los grupos de productos, elementos o categorías, conducente a nuevas ideas en el ámbito de la gestión, como la gestión de recursos humanos, comportamiento organizacional, el espíritu empresarial y la gestión estratégica, aportando un nuevo enfoque metodológico en la investigación de gestión.

El trabajo de Barnett (2007) refleja una variación entre las empresas y el entorno y dentro de las empresas en base a su capacidad por utilizar la RSC para mejorar las relaciones de rentabilidad, además trata de hacer que el modelo de negocio para la responsabilidad social empresarial sea específico de la empresa y no universal. En esta línea, Martin (2002) presenta la matriz de la virtud, como herramienta para ayudar a los ejecutivos a analizar la responsabilidad empresarial por considerarla un producto o servicio, proporcionando una manera de evaluar estas fuerzas y cómo interactúan. 
Recientemente, los últimos estudios de Vallester et al. (2012) suscriben la idea de que la RSC es, para las empresas que van más allá de las obligaciones legales y sus propios intereses, un mecanismo para abordar y gestionar el impacto que sus actividades tienen sobre la sociedad y el medio ambiente.

Todos estos trabajos indican que la RSC influye y afecta a todos los grupos de interés en todas las actuaciones de la empresa y su entorno. Por tanto, identificar los grupos de interés, y cuáles son sus expectativas, es un aspecto que facilita la implantación integral de la RSC en el seno de cualquier organización. Esto permitirá a las empresas atender correctamente las necesidades de sus grupos de interés y planificar las actuaciones de responsabilidad social que llevarán a cabo con los mismos.

\subsubsection{Disposición Social al cambio}

Aquí se recogen aquellos trabajos en los que el entorno social actúa como facilitador de la RSC. Para ello las organizaciones deben estar dispuestas no sólo a cambiar, sino a implantar y ejecutar esos cambios que conlleven mejoras sociales en todas las actuaciones empresariales y que afectan a todos los grupos de interés.

\subsubsection{Cambio Social}

La aplicación de actuaciones socialmente responsables por parte de las empresas influye y cambian el entorno y la propia empresa, teniendo en cuenta que la gestión empresarial es lo suficientemente difícil para los directores generales como para tener que plantearse cambios a diario para que sus empresas funcionen en el largo plazo. Así por ejemplo, Lévy et al. (2007), ponen de manifiesto cómo grandes empresas están luchando por conseguir mejoras, no sólo para su empresa, sino también para su entorno, de manera responsable. Como indican Scherer et al. (2013), los problemas en relación con la producción, distribución y consumo de bienes y servicios desafían cada vez más la legitimidad de las empresas.

Con la evolución y cambio en las organizaciones en las últimas décadas, la filantropía corporativa se ha convertido en una parte integral de la estrategia empresarial. Windsor (2006) indica que existe cierta tendencia a un aumento del voluntarismo. Además, argumenta que la fuerza de la economía no debe basarse en unas expectativas poco razonables sobre la moral de los agentes del mercado, sino que todos los agentes del mercado deben practicar una moral imparcial. Sin embargo, Hess et al. (2002) identifican una forma emergente de participación de la comunidad empresarial denominada "Iniciativa Social Corporativa" (CSI). Estos programas se diferencian de sus predecesores en que están conectados a los valores fundamentales de la empresa, que responden a las presiones morales, basadas en las competencias básicas de la empresa, y tienen claros los objetivos y los medios de medición.

El rendimiento de una organización va a depender de su capacidad y facilidad de adaptarse a los cambios que según las necesidades requieran sus grupos de interés. En función de la adapta- 
ción a esos cambios se obtendrá mayor rendimiento social y, por tanto, un aumento de los resultados empresariales, mayor ventaja competitiva y perdurabilidad en el tiempo de la empresa. Tal como indican Carmeli et al. (2007) es necesario un correcto ajuste en la identificación de los cambios y las implicaciones que conllevan en el rendimiento de la organización para unos óptimos resultados. En esta línea de investigación, Höllerer (2013) contribuye con un trabajo que examina la interpretación de los temas y aspectos sociales con el fin de estudiar la adopción y difusión de los conceptos de organización y prácticas de gestión.

\subsubsection{Actitud Social}

Desde hace aproximadamente una década estamos viviendo la transición de una economía basada en los bienes a una economía basada en los flujos de información, lo que ha creado importantes retos para el diseño de las organizaciones por el desencadenamiento de cambios en la organización. En este bloque se refleja la necesidad de favorecer los cambios, teniendo en cuenta que las formas de organización en nuestra economía han cambiado. Child y Gunther (2001) consideran que las nuevas formas de organización resultantes deben hacer frente a cuatro temas centrales: la interdependencia, lo inmaterial, la velocidad y el poder. Las investigaciones en esta línea proporcionan evidencias de cómo las formas han cambiado, así como qué aspectos de la teoría tradicional es probable que sigan siendo fuertes. Margolis y Walsh (2003) analizan cómo la teoría de la organización y la investigación empírica han respondido a la tensión sobre la participación de las empresas en la vida. Proponen que las empresas deben hacerse eco de las necesidades sociales que se encuentran en su comunidad, en su medio cercano, y deben tomar medidas para que esas necesidades queden cubiertas.

Una de las razones por las que es tan difícil definir la Responsabilidad Social Corporativa es que lo que se considera un comportamiento responsable de las empresas cambia con el tiempo. Según Rivoli y Waddock (2011), estamos ante un término dinámico y en evolución, por tanto, ya sean normas de comportamiento o leyes, las empresas se ven presionadas a adoptar comportamientos adicionales y mejorar los existentes en cuanto a responsabilidad corporativa. Teniendo en cuenta que las empresas se mueven en entornos dinámicos, Salge y Vera (2013) examinan los antecedentes, consecuencias y moderadores de las capacidades de aprendizaje incrementales, entendida como la capacidad de una organización para adaptarse gradualmente y ampliar su base de conocimientos, y sugieren un aprendizaje gradual que estará relacionado positivamente con el rendimiento organizacional.

Los trabajos de Mena y Waeger (2014) y de Waddock (2008) ponen de manifiesto la rápida evolución de las nuevas infraestructuras que están presionando a las empresas a ser más responsables, como el Global Reporting Initiative. En este sentido los ejecutivos a cargo de la responsabilidad social de las empresas deben insistir en medidas claramente definidas de éxito. Las empresas que crean puestos de trabajo estables y productivos, no explotadoras y las vibrantes economías locales son el único programa sostenible para la reducción de la pobreza masiva (Beck y Ogden, 2007). Con la apli- 
cación de medidas de este tipo se estarían introduciendo de manera indirecta, y desde instancias políticas y gubernamentales, medidas de responsabilidad social que vendrían a mejorar la actitud social a los cambios y a la aplicación de medidas socialmente responsables.

\subsubsection{Contexto geográfico}

No es igual la aplicación de la RSC en todos los países, ni en distintas zonas de los mismos países. Las investigaciones de Matten y Moon (2008) abordan esta cuestión de cómo y por qué la RSC es diferente entre países y cómo y por qué cambia. Así mismo, Bondy y Starkey (2014) plantean que las estrategias de internacionalización integradas no resuelven los problemas globales y locales en materia de responsabilidad social. En este apartado reflejamos estudios de casos en empresas y países, y encontramos ejemplos de comparativas entre empresas socialmente responsables y entre distintos países.

\subsubsection{Localización empresarial}

Warren y Tweedale (2002) revisan los progresos realizados en el Reino Unido en la evolución de la historia de los negocios y la adopción de la ética empresarial en la enseñanza universitaria. En este mismo país, años más tarde, Sharratt et al. (2007) tratan la imposición de obligaciones sociales en la industria del suministro de energía ofreciendo una oportunidad para examinar la forma en que se interpreta la responsabilidad social de las empresas y el alcance e influencia de un compromiso con el regulador.

George (2014) realiza una investigación en empresas estadounidenses sobre la influencia de la política y los sistemas económicos en las desigualdades sociales y empresariales, identificando las condiciones en las que las organizaciones infligen menos daño y alivian más el sufrimiento. Doh y Guay (2006), encuentran que las diferentes estructuras institucionales y legados políticos en los EE.UU. y la UE son factores importantes para explicar cómo los gobiernos, las ONG y la comunidad política más amplia determinan e implementan la RSC en las preferencias con respecto a estas dos regiones del mundo.

Arya y Zhang (2009) realizan un estudio sobre las empresas que han alineado sus estrategias a las nuevas regulaciones sociales instituidas en África del Sur, identificando que las empresas que llevan a cabo acciones socialmente responsables obtienen unos resultados financieros sustantivamente mayores, y en rentabilidad para los accionistas significativamente más altos, que aquellas empresas que no las aplican. Sin embargo, Surroca et al. (2013) explican cómo la presión de los grupos de interés en el país de una empresa multinacional lleva a la transferencia de prácticas socialmente irresponsables de su sede a sus filiales en el extranjero. En la misma línea de estudios sobre empresas multinacionales, Bondey y Starkey (2014) ponen de relieve las preocupaciones sobre la cultura nacional de empresas extranjeras y temas locales de la empresa y sugieren que las estrategias de internacionalización integradas no resuelven los problemas globales y locales en materia de RSC. Bailey 
y Spicer (2007) exploran el grado de convergencia frente a la divergencia en las actitudes y el comportamiento previsto en relación con la ética empresarial entre personas de diferentes nacionalidades.

Así pues, la aplicación de medidas y actuaciones de responsabilidad conllevan generación de beneficios no sólo económicos. La RSC no es algo aislado, depende de otros factores, como la zona geográfica, gobierno del país, tipo de empresa, otros grupos de interés y resto del entorno.

\subsection{Gestión estratégica de la Responsabilidad Social Corporativa}

En este apartado se recoge la importancia de utilizar una visión estratégica a la hora de implantar RSC en la empresa y por ende en diferentes sectores. Se contemplan numerosos problemas teóricos y empíricos no resueltos, relacionados con las implicaciones estratégicas de la RSC. Esto incluye la definición de Responsabilidad Social Corporativa; las diferencias en la identificación institucional en materia de RSC entre los países; la determinación de las motivaciones para la RSC, describiendo las estrategias de responsabilidad; la modelización de los efectos de la responsabilidad social en la empresa y en los grupos de interés, determinando los efectos del liderazgo y la cultura empresarial en las actividades de responsabilidad y evaluando los efectos de la responsabilidad en las empresas y los grupos de interés que miden la demanda de la RSC; la medición de los costes y la evaluación de la actual base de conocimientos con respecto a RSC (McWilliams et al., 2006).

\subsubsection{Adopción de la Responsabilidad Social Corporativa}

En este apartado se tratan aquellos trabajos que se centran en describir los procesos previos a la implantación, es decir aquellas actuaciones que la empresa debe plantearse para llevar a buen término las políticas y hábitos de RSC.

\subsubsection{Planificación y Organización del Proceso de Adopción}

Para algunas empresas la RSC puede tener una gran influencia en la estrategia corporativa. Las compañías que hacen un compromiso sustancial con la RSC es probable que encuentren que esto implica desafíos importantes con respecto a la formulación y puesta en práctica de la estrategia de RSC, debido a la incertidumbre que inevitablemente conlleva asociada con la firme determinación de las obligaciones sociales (Smith, 2003).

Husted y De Jesus Salazar (2006), indican que en la estrategia empresarial se utilizan herramientas de microeconomía para definir el nivel óptimo de producción social que se debe conseguir. Estos autores demuestran que es más prudente para que la empresa actúe estratégicamente estar obligada a realizar inversiones en responsabilidad social corporativa. 
Un ejemplo de diseño organizacional lo encontramos en la empresa Nike. Zadek (2004) describe el camino que Nike ha recorrido para llegar a un lugar mejor éticamente, cultivando y defendiendo las prácticas empresariales responsables.

Otro de los pilares son los directivos o líderes empresariales. En este sentido McWilliams et al. (2006), sugieren que la responsabilidad social es una ventaja ejecutiva, en el sentido de que los gerentes usan la RSC para avanzar en sus carreras o agendas personales. Sin embargo, Nonaka y Takeuchi (2011), comentan que en una era de creciente discontinuidad muchos líderes tienen dificultades para reinventar sus empresas con la suficiente rapidez para hacer frente a las nuevas tecnologías, los cambios demográficos y las tendencias de consumo. Porter y Kramer (2006) introducen un marco de trabajo que las empresas pueden utilizar para identificar las consecuencias sociales de sus acciones, para determinar qué iniciativas de RSC deben desarrollarse y para encontrar más formas eficaces de hacerlo. En suma, proponen un enfoque estratégico para la participación de las empresas en la sociedad. En esta línea, los estudios de Galbreath (2010) indican que los esfuerzos por una planificación estratégica formal están positivamente relacionados con la RSC, es decir la responsabilidad forma parte de la corriente principal de la empresa. Una línea de análisis anterior, planteada por Aguilera et al. (2007) sugiere que los gerentes no deben ver la RSC como un lugar externo "add-on", sino como una importante herramienta de gestión. Igualmente, Berger, Cunningham y Drumwright (2007) investigan cómo la RSC se introduce en el día a día de la cultura, los procesos y actividades de una empresa, examinando cómo se justifica la inversión en actividades de RSC. Por su parte, Karnani (2011a) se basa en la afirmación de que las empresas tienen la responsabilidad social para alcanzar algunos objetivos sociales más amplios y pueden hacerlo sin un sacrificio financiero excesivo, si se centran en una buena organización pueden "hacerlo bien haciendo el bien". En este sentido, en investigaciones posteriores Raffaelli y Glynn (2014) afirman que la infraestructura e identidad organizacional afectan significativamente la práctica de adopción de RSC; así mismo Den Hond et al. (2014) argumentan que la RSC de la empresa es una actividad que debe estar inmersa en la política de la empresa. En base a estas investigaciones se puede afirmar que la adopción de prácticas de RSC en las organizaciones debe ser planificada y estar inmersa en la gestión y organización empresarial.

\subsubsection{Implantación de la Responsabilidad Social Corporativa}

En este apartado se agrupan todos aquellos trabajos que indican cómo introducir hábitos, maneras, actitudes de RSC en las empresas, mercados y sectores una vez realizada la planificación y organización empresarial.

\subsubsection{Empresas, sectores y mercados}

En este apartado se expone un modelo de diseño organizativo con sentido de responsabilidad, un proceso en el que las decisiones en la organización se tomen con sentido y se presenta como se introducen las empresas en sus mercados de actuación y por ende como se debería trabajar con sentido de responsabilidad social en los distintos sectores en los que operan. 
Para Campbell (2007) algunos estudiosos del ámbito de la administración han dirigido su atención a investigar el papel de las instituciones para permitir y restringir el comportamiento socialmente responsable en las empresas. El examen de la extensa literatura de la Responsabilidad Social Corporativa revela que las empresas adoptan políticas de responsabilidad social con el fin de asegurar la legitimidad o diferenciación competitiva (Waddock y Graves, 1997; Bansal y Hunter, 2003).

En los últimos años las empresas han comenzado a reconocer la importancia de la RSC, aun teniendo en cuenta la reciente oleada de escándalos empresariales, fraudes contables y dudosas prácticas en los negocios. Investigaciones como la de Christensen (2014) reafirman estas ideas, pues su trabajo se fundamenta en la responsabilidad y la irresponsabilidad social corporativa, centrándose en cómo los líderes influyen en ambas actividades. El clima actual de mayor escrutinio hacia un comportamiento corporativo (Waddock, 2000; Raar, 2002) pone de relieve, tal vez como nunca antes, la necesidad de una solidez conceptual para guiar los compromisos de RSC llevados a cabo por las empresas (Basu y Palazzo, 2008).

En los últimos años se pretende explicar cómo la liberalización del mercado está obligando a los ejecutivos de negocios y activistas sociales a trabajar juntos (Brugmann y Prahalad, 2007). Así, la investigación de Mackey et al. (2007) indica que para comprender la relación entre la RSC y el valor de mercado de la empresa, es fundamental examinar las condiciones de la oferta y la demanda de oportunidades de inversión en empresas que adoptan el cambio social. Ross (2014) proporciona un tratamiento matemático formal de la relación entre la ventaja competitiva y el riesgo en la interacción estratégica argumentando otros dos aspectos importantes del mercado, la rivalidad del número de empresas competidoras y la de su mercado de productos.

En investigaciones posteriores, Lindgreen y Swaen (2010) argumentan que la implantación de actuaciones y hábitos socialmente responsables requiere sensibilidad a las normas y valores de las comunidades de acogida, así como conversaciones abiertas con los representantes empresariales y resto del entorno. Igualmente, las investigaciones de Maon et al. (2010) indican que sobre la base de una concepción orientada a los grupos de interés de la responsabilidad social se ofrece una perspectiva dinámica multidimensional que integra los aspectos morales, culturales y estratégicos del proceso de desarrollo de la RSC y sus implicaciones organizativas.

En la era de las tecnologías es importante destacar la innovación en el diseño del modelo de negocios actual, que está atrayendo cada vez más atención de las empresas. A través de procesos sistemáticos de múltiples configuraciones de diseño para los modelos de negocio y el uso de protocolos de decisión para elegir el diseño que tenga una alta probabilidad de éxito (Chatterjee, 2013).

Estas investigaciones vienen a confirmar que todo proceso empresarial que pretenda contar con actuaciones socialmente responsables debe contar previamente con un proceso y orden lógico de planificación y organización. 


\subsection{Impacto de la Responsabilidad Social Corporativa}

En este apartado se hace referencia a la utilidad que tiene la RSC para la empresa en sus diferentes ámbitos: social, medioambiental y económico. Agrupados, por una parte, en base a la aplicación de valores y al incremento de los mismos en la organización y, por otra, en función de los resultados empresariales y ventaja competitiva que generan para la empresa.

\subsubsection{Nivel social}

Toda empresa que se considere responsable socialmente debe y tiene que tener sentido ético, unas mínimas normas y valores en su desarrollo empresarial. Así mismo estas empresas deben ir incrementando sus valores y aplicándolos en su entorno y a las actuaciones empresariales diarias, considerando los recursos humanos como el pilar fundamental de su estructura y, por tanto, contribuyendo de forma responsable a su cuidado. Se tratan también en este apartado, las normas y actitudes de respeto y atención al medioambiente como parte del entorno empresarial.

\subsubsection{1. Ética empresarial}

La literatura de la ética empresarial contiene una serie de áreas relativamente distintas de investigación. Para Oosterhout et al. (2006), la teoría integrativa es sin duda la teoría más prometedora de la ética empresarial hasta la fecha. Según Bartlett (2003) destacan tres áreas principales: las teorías filosóficas de la ética; el desarrollo cognitivo y moral y los valores del trabajo, el razonamiento moral y la ética en la toma de decisiones.

Para Welsh y Ordoñez (2014), la investigación en el campo de la ética se ha visto afectada por decisiones racionales y deliberadas. Sin embargo, para Weaver et al. (2014) el pensamiento ético se encuentra impulsado por la intuición. Bos y Willmott (2001) plantean si es posible la ética empresarial no sólo para desafiar una jerarquía empresarial sino para evitar su retroceso. Para Jacobs (2004) la integridad es un concepto fundamental en la ética empresarial. Sin embargo, no todos los autores hablan de ética en sentido positivo, también encontramos estudios como los de Kaptein (2008) que hablan de comportamientos poco éticos en el lugar de trabajo. Contu (2014) continúa con la investigación relativa a la realización de prácticas irregulares o poco éticas en el ámbito de la empresa, realizando informes para combatir las prácticas ilegales. Barraquier (2011) se centra específicamente en los beneficios y el hecho de conjugar la ética en los negocios para generar beneficios empresariales y Lllia et al. (2013), se centran igualmente en los resultados empresariales, en la comunicación y transparencia de los resultados como parte del proceso de política corporativa. En relación con los resultados empresariales la investigación de Chin et al. (2013), indica que la ideología política de los CEO's influye en las prácticas y políticas de RSC en la empresa.

Los estudios de gestión empresarial tratan la ética como parte del tejido empresarial y en este sentido Clegg et al. (2007) desarrollan una conceptualización de la ética empresarial como una práctica 
partiendo de elecciones morales. Por su parte, De Cremer et al. (2011) dicen que la administración pública y las empresas, en general, están constantemente enfrentando importantes desafíos éticos, de ahí que los estudios y aplicación de normas y conductas éticas sean tan importantes. En esta línea, los estudios de Kleyn et al. (2012), hablan de una construcción de la identidad ética corporativa, un proceso conocido como "eticalización".

Las investigaciones de Trevino et al. (2006) confirman la importancia del comportamiento ético de una organización. Siguiendo esta línea el trabajo de Godfrey y Mahoney (2014) ratifica el trabajo previo de Barnard (1938) sobre la dimensión moral de la organización. Considerando incluso la práctica de los gerentes de ignorar o hacer la vista gorda ante las amenazas o irregularidades que en última instancia ponen en peligro sus negocios (Bazerman, 2014).

En definitiva los resultados empresariales vendrán ligados a las actuaciones de política corporativa de la empresa y contempladas, entre ellas, las actuaciones éticas empresariales en todos los ámbitos de la empresa.

\subsubsection{Incremento de Valores}

En este apartado se pretende ayudar a todos los grupos de interés a lidiar con los problemas morales de los negocios, o cómo indica Barnett (2014) porqué algunos grupos de interés ignoran las malas conductas de las empresas, instando a los esfuerzos para desarrollar una adecuada "estrategia moral administrativa" (Soule, 2002). La confianza que se va a crear en la empresa, y fuera de ella, tiene que comenzar en la propia empresa, llegando a aprender que si no puede ser un ejemplo de lo que quiere ser, la organización no tendrá ninguna posibilidad de convertirse en una "empresa transparente". La base de una empresa verdaderamente responsable es la confianza (Hollender, 2004). Sin embargo, y como demuestra la investigación de Cording et al. (2014), la falta de autenticidad de la organización hacia empleados y clientes se asocia con una menor productividad.

Las investigaciones de Waldman et al. (2006) consideran los valores en las organizaciones con respecto a los directivos empresariales, haciendo referencia a las cualidades morales y éticas de los líderes carismáticos. Un buen ejemplo de aplicación de valores lo encontramos en Berry y Robinson (2009) en la que se trata el desarrollo de un sistema de telecomunicaciones que utiliza la tecnología de reproducción de audio y vídeo para que las personas sordas aprendan a comunicarse con las personas no sordas.

Según Eccles et al. (2007), en los últimos años las investigaciones han girado en torno a la evaluación y gestión de los riesgos en una amplia gama de áreas, desde los precios de los productos a los desastres naturales. En contra de estos estudios surgen los de Crane et al. (2014), que critican el concepto de creación de valor defendido por Michael Porter y Mark Kramer en investigaciones previas. 
Los estudios de Janney y Gove (2011) indican una estrecha relación entre RSC y gestión de la reputación. Sin embargo, para muchas empresas, el activo más valioso radica no en el balance ni en el capital humano de la fuerza de trabajo, sino más bien en su reputación (Minor y Morgan, 2011). Puede este ser el motivo por el cual los diferentes grupos de interés ignoren las malas conductas de las empresas (Raffaelli y Glynn, 2014). Por ejemplo, la reputación de IBM para ser un proveedor de soluciones empresariales le ha permitido superar a sus rivales durante muchos años. Para McDonald, su reputación es ser un lugar económicamente ideal para comer las familias, lo que ha mantenido su cuota de mercado frente a la feroz competencia de otras cadenas (Minor y Morgan, 2011).

Si bien existe un amplio apoyo a la idea de que las organizaciones con mejor reputación superan a sus rivales, existe incertidumbre acerca de cómo crear la reputación, especialmente entre los directivos responsables de esta tarea. Según Dowling y Moran (2012), una reputación basada en la estrategia de la organización tiene una mejor oportunidad de proporcionar una ventaja competitiva sostenible y es menos reproducible por la competencia. Según Eccles et al. (2007), son los directivos los que hacen un mejor trabajo para evaluar las amenazas existentes y potenciales a la reputación de sus empresas y decidir si aceptan un riesgo particular o toman medidas para evitarlo o mitigarlo.

En otra línea, Lange y Washburn (2012) centran sus estudios en conductas irresponsables como uno de los valores menos apreciados y más criticados por los consumidores y resto de grupos de interés.

Otro de los valores a destacar de las empresas socialmente responsables es su efectividad, la empresa trata de alcanzar metas y resultados que van más allá del valor de las acciones o beneficios económicos. Las decisiones de la empresa se hacen en términos de distribución de rentas y sus inversiones y actividades pueden ser consideradas o identificadas como socialmente responsables 0 irresponsables. La dificultad es estar dispuestos a aceptar el carácter bueno y malo de la organización. El objetivo de la empresa es ser guiado por la necesidad de utilizar la RSC para "bueno" por sí sola (Devinney, 2009).

Según las investigaciones llevadas a cabo la aplicación de valores en la empresa en todas sus vertientes, de manera positiva o negativa, lleva implícita una respuesta empresarial y consecuentemente de todos los grupos de interés, reputación, acciones socialmente responsables, irresponsabilidad, efectividad, ética empresarial. Todos ellos, valores a aplicar o mejorar en empresas que se consideren socialmente responsables. Sin duda la aplicación de los mismos redundará no sólo en ventaja competitiva, sino en mayor rendimiento financiero $y$, en definitiva, en valores para generar valor empresarial. 


\subsubsection{Recursos Humanos}

Los recursos humanos de la empresa son uno de los pilares más importantes en las organizaciones, y hoy en día las investigaciones demuestran que los empleados prefieren trabajar para empresas socialmente responsables aunque perciban salarios inferiores a la media, pues esto lo ven compensado en otros ámbitos como puede ser el de la formación, beneficios sociales, o una mayor estabilidad en los puestos de trabajo.

Ejemplos de buenas prácticas con trabajadores y proveedores se encuentran en los estudios de Frenkel y Scott (2002), en relación con la empresa ADIDAS, en el que la empresa muestra el establecimiento y mantenimiento de normas laborales para los empleados de los contratistas en los países donde los trabajadores tienen poca protección, o ninguna, contra la explotación.

Los estudios de Bhattacharya et al. (2008) indican que a medida que los dirigentes empresariales se dan cuenta de la importancia de los recursos humanos, crecen y se intensifican también las actividades de responsabilidad social, pues comprenden un medio legítimo y convincente para atraer y retener a los buenos empleados. En estudios más recientes, Mirvis (2012) analiza, igualmente, la importancia de la responsabilidad social corporativa para los empleados, incluyendo su impacto en la motivación, la identidad y sentido de significado y propósito.

En otra línea, las investigaciones de Muthuri et al. (2009) hablan de empleados voluntarios, considerados cada vez más como un medio para mejorar la comunidad de las empresas y las relaciones laborales. Estos autores investigan la contribución de los empleados voluntarios a la responsabilidad social de las empresas. En particular, cómo contribuyen al capital social, las relaciones sociales y los recursos que sustentan la creación de capital social, así como su contribución en el programa general de responsabilidad social corporativa de las empresas.

\subsubsection{Nivel económico}

En este apartado se tratan aquellos trabajos que analizan los resultados empresariales y la ventaja competitiva para las empresas que trabajan en Responsabilidad Social Corporativa, y cómo tras la aplicación y puesta en práctica de todos los mecanismos anteriores se llega a obtener mayor rendimiento financiero y a ser más competitivas en el mercado.

\subsubsection{Resultados empresariales}

En este apartado se presentan los efectos sobre las empresas y el entorno, ya sean positivos 0 negativos, del uso y aplicación de actuaciones responsables.

Algunos estudios muestran que hay empresas que tienen dos objetivos: la maximización de los beneficios y el rendimiento social. Así, Porter y Kramer (2002) analizan en grandes empresas de 
Estados Unidos actividades filantrópicas durante los últimos 15 años, contemplando que los ejecutivos cada vez más se ven a sí mismos como atrapados entre los críticos que exigen mayores niveles de "responsabilidad social corporativa" y los inversores que aplican presión para maximizar beneficios a corto plazo. Por tanto, la adopción de un enfoque centrado en el contexto va en contra de la práctica filantrópica actual y requiere un enfoque mucho más disciplinado que prevalece en la actualidad y que puede hacer que las actividades de la empresa sean mucho más eficaces.

Mintzberg et al. (2002) hablan de prosperidad real en diferentes países desde Estados Unidos a América del Sur y África, indicando que se debe combinar el desarrollo económico con la generosidad social y que se requiere una nueva filosofía de compromiso social y de gestión. De igual modo, Deckop (2006) indica, a través de una muestra de 313 empresas estadounidenses de la lista Standard \& Poor 500 , que el rendimiento social de las empresas (CSP) se considera cada vez más un resultado de negocio importante para los investigadores, inversores y la sociedad en su conjunto. Estas investigaciones, junto con las de Tang et al. (2012) y las de Schuler y Cording (2006), muestran que el rendimiento social de la empresa se relaciona positivamente con el rendimiento financiero. Igualmente los argumentos de Du et al. (2010), en base a la comparación de varios estudios previos, señalan que la participación empresarial en actividades de responsabilidad social empresarial no sólo puede generar actitudes favorables y mejores comportamientos de apoyo sino también, a largo plazo, crear imagen corporativa, fortalecer las relaciones y los intereses de la compañía y mejorar los comportamientos de todos los grupos de interés. Por su parte Peloza y Falkenberg (2009) piensan que los directivos deben hacer coincidir las estrategias de responsabilidad con los objetivos de la empresa. La investigación llevada a cabo por Oikonomou et al. (2014), centrada en una muestra de 769 empresas estadounidenses que cotizan en el índice Standard \& Poor 500 para los años comprendidos entre 1991 y 2008, indican que las empresas normalmente presentan un cuadro mixto de rendimiento social con indicadores positivos y negativos exhibidos por la misma empresa. Igualmente, según el estudio de Wang y Choi (2013), centrado en una muestra de 622 empresas de la base de datos KLD, demuestra que el establecimiento de buenas relaciones con los grupos de interés está influenciado no sólo por conseguir un alto nivel de rendimiento financiero, sino también por su capacidad para ofrecer rendimiento social eficiente. Por lo tanto, el nivel y consistencia en el rendimiento social de las empresas deben tener repercusiones financieras significativas.

Pearce y Doh (2005), en base a diferentes estudios cuantitativos centrados en casos de grandes empresas, muestran que las empresas pueden combinar las iniciativas de responsabilidad social con un rendimiento justo a sus propietarios y el uso eficiente de los recursos llegando a la conclusión de que es posible combinar la contribución al bien común con el avance de los amplios objetivos financieros y de comercialización. Igualmente, Doh et al. (2009), utilizando información para el período 200005 de las bases de datos Carvert, Domini, COMPUSTAT y KLD, ponen de manifiesto la delicada interacción entre las diferentes evaluaciones de rendimiento social, la reputación y las medidas de rendimiento financiero y operativo. Por su parte, Shuler y Cording (2006) presentan un modelo en el que relacionan rendimiento social de las empresas y rendimiento financiero corporativo (PPC), con el modelo de comportamiento de compra de los consumidores. 
Frente a estas investigaciones que establecen la correlación entre rentabilidad social y financiera, aparecen las investigaciones de Vogel (2005) y Karnani (2011b) que, analizando la investigación empírica sobre responsabilidad social, plantean el debate sobre si alguna actividad socialmente deseable es rentable, siendo entonces descrita como una operación inteligente para los negocios. Así mismo, Chun et al. (2013), a partir de una muestra de 263 empresas coreanas en 2008, destacan la importancia de la aplicación de prácticas éticas corporativas en el rendimiento financiero de la empresa.

No se puede concluir esta agrupación sin nombrar el "efecto Wal-Mart", en este caso atendiendo a una publicación de Freeman (2006) en la que sostiene que el debate sobre el enfoque empresarial de los precios bajos y las lagunas de impacto social y económico ponen de relieve la comprensión contemporánea de los negocios, donde las relaciones de la empresa con clientes, proveedores, empleados, comunidades e inversores se consideran importantes para el proceso de creación de valor y mejora de los resultados empresariales, lo que obliga a mantener la gestión de sus intereses en esta línea.

En definitiva todas las investigaciones nos indican que la aplicación de hábitos, conductas y valores de Responsabilidad Social Corporativa redundará en resultados empresariales positivos, a nivel operativo, financiero y social.

\subsubsection{Ventaja competitiva}

Para la mayoría de las empresas, el diseño de la organización no es ni una ciencia ni un arte, es un oxímoron. Aunque la mayoría de los ejecutivos se dan cuenta cuando sus diseños organizacionales no están funcionando bien, pocos toman medidas significativas, en parte porque carecen de un marco práctico para guiarlos. Goold y Campbell (2002) proporcionan ese marco; presentan nueve pruebas que pueden utilizarse para evaluar o diseñar la organización existente o crear uno nuevo, generando con ello mayor ventaja competitiva para la organización.

Teniendo en cuenta que las empresas tienen dos objetivos: empresariales y sociales, una importante pregunta sin respuesta es si la capacidad dinámica general engendra competencias en estas dos áreas. Marcus y Anderson (2006) realizaron un estudio de la industria de alimentos al por menor en EE.UU., encontrando que mientras que una capacidad dinámica general afecta a la competencia de las empresas en la gestión de la cadena de suministro (una competencia de negocios), no afecta a su competencia en la gestión del medio ambiente (una competencia social). Estos resultados sugieren que los factores que impulsan la ventaja competitiva no son los mismos que los que impulsan la responsabilidad social.

Sin embargo, McWilliams y Siegel (2011) analizan la creación y captura de valor privado y social de las empresas que adoptan responsabilidad social corporativa, definida como una actividad "responsable" que permite a una empresa lograr una ventaja competitiva sostenible, sin importar el motivo, donde RSC se refiere a "la provisión privada de bienes públicos". En esta misma línea, la investiga- 
ción de Orlitzky (2013) indica que controlar el mercado está percibido como socialmente responsable y hace que las empresas generen más valor para sus empresas. Delios (2010) indica que son los líderes de la organización los que deben cambiar la forma de hacer la gestión empresarial, pudiendo llevarlo a cabo a través de su influencia sobre los responsables políticos y otras organizaciones que dan forma a las normas formales e informales de las prácticas empresariales en todas las regiones del mundo, generando así mayor ventaja competitiva.

\subsubsection{Nivel medioambiental}

En este apartado se tratan aquellos trabajos que analizan la sostenibilidad ambiental y los resultados medioambientales para las empresas que aplican normativa legal al respecto o que trabajan en Responsabilidad Social Corporativa.

\subsubsection{Sostenibilidad}

Buena parte de los valores de una empresa se transmiten a su entorno a través de una sólida reputación. Si es ampliamente reconocida será el activo más valioso de una empresa convirtiéndose la sostenibilidad en un componente importante de la reputación corporativa. Muchos grupos de interés, desde los clientes, inversores, empleados, gerentes de compras, informan que la sostenibilidad y el medioambiente son factores importantes en sus procesos de decisión. A menudo existe una brecha importante entre percepciones de los interesados y el rendimiento de la empresa. Sin embargo, las empresas que integran la sostenibilidad en su cultura y sus prácticas de negocios son más capaces de integrarla en todo su ámbito de actuación y entorno (Peloza et al., 2012).

Por su parte, las investigaciones de Kock et al. (2012) exploran el impacto de mecanismos de gobierno corporativo en su desempeño ambiental. Flammer (2013) examina si los accionistas son sensibles a la huella ambiental de las empresas. Igualmente las investigaciones de Lampikoski et al. (2014) sugieren formas de ayudar a los administradores a superar barreras e incorporar tres funciones de gestión en su agenda y cultura corporativa para promover la sostenibilidad empresarial.

Algunos años antes, las investigaciones de Brammer y Pavelin (2006) muestran la necesidad de ajuste entre los grupos de interés para un correcto rendimiento social de las empresas y un sólido historial de rendimiento ambiental, pudiendo mejorar o dañar la reputación empresarial, dependiendo de si las acciones de la empresa se ajustan o no con las preocupaciones ambientales del resto del entorno. La investigación de Delmas et al. (2013), relativa a la aparición de inversión socialmente responsable, explora la relación entre el desempeño ambiental y financiero.

En las investigaciones analizadas se deduce la importancia que desde siempre se le ha dado al aspecto medioambiental de la RSC. No obstante, puede verse que en los últimos tiempos los estudios en este aspecto han descendido en buena parte, centrándose más en aspectos de rendimiento y resultados. 


\section{5.- Conclusiones y líneas de investigación futuras}

En este trabajo se realiza una agrupación de la literatura que ayuda a cualquier persona o estudioso que quiera iniciarse en RSC para saber qué es lo que se ha investigado en RSC a nivel organizativo, centrado en tres aspectos fundamentales: La influencia del entorno social, donde se analiza la adopción de actitudes y comportamientos de responsabilidad social en las empresas. La gestión estratégica de la empresa, donde se analiza la importancia de utilizar una visión estratégica a la hora de implantar RSC en la empresa. Y finalmente el impacto que supone la aplicación de actuaciones de Responsabilidad Social Corporativa, haciendo referencia a la utilidad que tiene la RSC para la empresa en sus diferentes ámbitos: social, medioambiental y económico.

Este trabajo puede ser de utilidad para las empresas que quieran implantar RSC como sistema de gestión ya que ayudará a entender la adopción, implantación y mejora como empresa socialmente responsable. Partiendo de la influencia que ejerce el entorno y que se ejerce sobre el entorno de la empresa con sus actuaciones, cómo afecta a la sociedad y de qué manera se aceptan y aplican los cambios en función del área geográfica, influencia de los gobiernos, entorno social y ambiental, etc. Igualmente, se describe el proceso para llevar a cabo las políticas y hábitos de RSC en la empresa, cómo se fijan los objetivos y cómo se deben alcanzar, cómo se lleva a cabo en la empresa y cómo se introduce en el mercado y consecuentemente en el sector empresarial del que se trate. Finalmente, se estudia la utilidad y práctica en cada uno de los niveles o ámbitos de aplicación de la RSC en la empresa y los resultados que de ello se derivan.

La agrupación aportada muestra que en Responsabilidad Social Corporativa se conjuga el compromiso social y organizacional. Planificar, organizar o diseñar un plan de actuación empresarial generará ventaja competitiva sobre otras empresas que no lo desarrollen. La evidencia empírica muestra que los mecanismos de gobierno corporativo como el consejo de administración, los incentivos empresariales, el mercado de control corporativo y el sistema legal y reglamentario determinan los niveles de rendimiento de las empresas.

Las interacciones entre las empresas y la sociedad y los cambios organizativos que se producen como resultado de la adopción de medidas de responsabilidad social de las empresas son inmensamente ricos y los fenómenos dinámicos, pero no han sido suficientemente explorados. Cómo indican las investigaciones de Vogel (2005), en los últimos 30 años de investigación no se ha encontrado ninguna relación causal definitiva entre RSC y resultados empresariales, siendo evidente que la investigación debe continuar en el futuro. Sin embargo, las investigaciones de Cheah et al. (2011) han demostrado que la relación existente entre ISR y rendimiento financiero es positiva o neutra, mientras que muy pocos han demostrado lo contrario. Por tanto, se hace necesario seguir investigando en este área para dotar de una base aún más sólida a unas conclusiones académicas ya de por sí válidas. 
En este trabajo queda plasmado que deben continuar tanto las investigaciones teóricas como empíricas debido a la necesidad de concretar un concepto de RSC, la necesidad de normativa reguladora desde instancias políticas y la necesidad de una medida que indique la aplicación de prácticas responsables en los diferentes sectores o zonas geográficas, así como demostrar que la aplicación de prácticas de Responsabilidad Social Corporativa mejoran los resultados empresariales y crean valor para la empresa.

La revisión de la literatura efectuada ha permitido identificar los siguientes retos para la investigación futura:

- Uno de los mayores retos a los que se está enfrentando la RSC es el de su medición. Este tema ya se comentaba en las investigaciones de Lindgreen y Swan (2010), Wood (2010) y Aguilera et al. (2007). Es difícil de cuantificar el valor de elementos que no tienen un importe económico objetivo como, por ejemplo, el saber hacer de los empleados o los resultados de investigaciones.

- Un importante reto a superar, referido a un prejuicio especialmente arraigado es el de la Inversión Socialmente Responsable (ISR), aquella inversión que busca financiar proyectos que no sólo sean rentables económicamente sino sostenibles medioambientalmente y justos socialmente; estos proporcionan un rendimiento financiero inferior al de la inversión no basada en tales criterios de responsabilidad.

- El consumidor final siente mayor aceptación por productos y servicios de empresas socialmente responsables e incluso está dispuesto a pagar un precio algo más elevado por esos productos o servicios, por ello sería preciso investigar si las empresas que realizan más campañas de marketing sobre sus actuaciones en responsabilidad social corporativa, también consiguen mejoras en sus resultados empresariales, sociales o medioambientales.

- La RSC es dinámica y cambiante y se aplica en todos los sectores empresariales, aunque su desarrollo e implantación difiere según la zona geográfica o en base al sector empresarial. En base a ello se detecta la necesidad de realizar investigaciones en diferentes sectores y en diferentes períodos.

- Surge la necesidad de concretar un concepto y normativa legal efectiva e implicación política. Cómo se viene tratando a lo largo de este trabajo y tal cómo reflejan las investigaciones de Lee (2008), Carroll y Shabana (2010), Scherer y Palazzo (2007 y 2011) y Aguilera et al. (2007), es necesario contar con un concepto único, unívoco y universal de RSC, que ante la multitud de estudios al respecto, y las acepciones del mismo, debería fijarse a instancias políticas y reglamentado correctamente, para que sea de aplicación universal y sin dar lugar a confu- 
siones. Asimismo, sería conveniente la aplicación de ciertas normas que, cómo en el ámbito medioambiental, impongan unas directrices básicas en la materia ${ }^{1}$.

- Se echan en falta prácticas de "benchmarking" en RSC, para intentar adoptar o copiar las prácticas de Responsabilidad Social Corporativa que mejor funcionen en empresas líderes. Por tanto, sería necesario realizar intercambios de conocimientos y experiencias de difusión de resultados.

Estos retos para la investigación futura se han planteado a raíz de una revisión de la literatura que ha estado acotada temporalmente y a las revistas de mayor impacto en el ámbito del Management. En este sentido, dichos retos surgen de la evaluación de la investigación publicada en 12 revistas de prestigio durante el periodo 2000-2014. Esta limitación en cuanto al ámbito de análisis provoca que sean necesarios estudios futuros que amplíen el alcance temporal y de revistas para validar la propuesta de retos a cubrir en un futuro.

\section{Agradecimientos}

Este trabajo ha sido financiado gracias al proyecto de investigación conseguido en la I Convocatoria de Ayudas de la Fundación BBVA a Proyectos de Investigación (Área de Socioeconomía).

\section{6.- bibliografía}

ABS, THE ASSOCIATION OF BUSINESS SCHOOLS (2010): "Academic Journal Quality Guide", Version 4. $\mathrm{Pb}$. The Association of Business Schools, United Kingdom.

AGUILERA, R.V., RUPP, D.E., WILLIAMS, C.A. \& GANAPATHI, J. (2007): "Putting the S back in Corporate Social Responsibility: A multinivel theory of social change in organizations", Academy of Management Review, 32 (3), 836-863.

AGUINIS, H. (2011): "Organizational responsibility: Doing good and doing well". In: S. Zedeck (Ed.), APA handbook of industrial and organizational psychology, Washington, American Psychological Association, 855-879.

1.- No obstante hay que reconocer los actuales avances de organismos e instituciones como la Comisión Europea y el Consejo Estatal de la Responsabilidad Social de la Empresa (CERSE) en esta materia, respondiendo al objetivo de agrupar en un mismo órgano a representantes de los distintos grupos de interés vinculados a la responsabilidad social de las empresas y asumiendo las competencias de acuerdo con el Real Decreto 343/2012 de 10 de febrero en España. 
AGUINIS, H. \& GLAVAS, A. (2012): "What We Know and Don't Know About Corporate Social Responsibility: A Review and Research Agenda", Journal of Management, 38 (4), 932-968.

AGUINIS, H., FORCUM, L.E. \& JOO, H. (2013): "Using Market Basket Analysis in Management Research", Journal of Management, 39 (7), 1799-1824.

ARYA, B. \& ZHANG, G. (2009): "Institutional Reforms and Investor Reactions to CSR Announcements: Evidence from an Emerging Economy", Journal of Management Studies, 46 (7), 1089-1112.

BAILEY, W.J. \& SPICER, A. (2007): "When does national identity matter? Convergence and divergence in international business ethics", Academy of Management Journal, 50 (6), 1462-1480.

BANSAL, P. \& CLELLAND, I. (2004): "Talking trash: Legitimacy, impression management, and unsystematic risk in the context of the natural environment", Academy of Management Journal, 47, 93103.

BANSAL, P. \& HUNTER, T. (2003): "Strategic explanations for the early adoption of ISO 14001", Journal of Business Ethics, 46, 289-299.

BARNETT, M.L. (2007): "Stakeholder influence capacity and the variability of financial returns to Corporate Social Responsibility", Academy of Management Review, 32 (3), 794-816.

BARNETT, M.L. (2014): "Why Stakeholders Ignore Firm Misconduct: A Cognitive View", Journal of Management, 40 (3), 676-702.

BARRAQUIER, A. (2011): "Ethical Behaviour in Practice: Decision Outcomes and Strategic Implications", British Journal of Management, 22 (s1), S28-S46.

BARTLETT, D. (2003): "Management and Business Ethics: A Critique and Integration of Ethical Decision-making Models", British Journal of Management, 14 (3), 223-235.

BASU, K. \& PALAZZO, G. (2008): "Corporate social responsibility: A process model of sensemaking", Academy of Management Review, 33 (1), 122-136.

BAZERMAN, M.H. (2014): "Becoming a first-class noticer. How to spot and prevent ethical failures in your organization", Harvard business review, 92 (7-8), 116-119, 131.

BECK, S. \& OGDEN, T. (2007): "Beware of Bad Microcredit", Harvard Business Review, 85 (9), $20-22$.

BENN, S. \& BOLTON, D. (2011): Key Concepts in Corporate Social Responsibility (SAGE Key Concepts series), Ed. Sage Publications Ltd., United Kingdom.

BERGER, I., CUNNINGHAM, P. \& DRUMWRIGHT, M. (2007): "Mainstreaming corporate social responsibility: Developing markets for virtue", California Management Review, 49 (4), 132-157.

BERRY, G.R. \& ROBINSON, P.B. (2009): "An Interview With James Lee Sorenson", Journal of Management Inquiry, 18 (4), 302-311. 
BHATTACHARYA, C.B., SEN, S. \& KORSCHUN, D. (2008): "Using Corporate Social Responsibility to Win the War for Talent", MIT Sloan Management Review, 49 (2), 37-44.

BONDY, K. \& STARKEY, K. (2014): "The dilemmas of internationalization: Corporate social responsibility in the multinational corporation", British Journal of Management, 25 (1), 4-22.

BOS, R. \& WILLMOTT, H. (2001): "Towards a Post-Dualistic Business Ethics: Interweaving Reason and Emotion in Working Life", Journal of Management Studies, 38 (6), 769-793.

BRAMMER, S. \& PAVELIN, S. (2006): "Corporate Reputation and Social Performance: The Importance of Fit", Journal of Management Studies, 43 (3), 435-455.

BRUGMANN, J. \& PRAHALAD, C.K. (2007). "New social compact", Harvard Business Review, 85 (2), 80-90.

CAMPBELL, J.L. (2006): "Institutional analysis and the paradox of corporate social responsibility". American Behavioral Scientist, 49 (7), 925-938.

CAMPBELL, J.L. (2007): "Why would corporations behave in socially responsible ways? An institutional theory of corporate social responsibility", Academy of Management Review, 32 (3), 946-967.

CARMELI, A., GILAT, G. \& WALDMAN, D.A. (2007): "The Role of Perceived Organizational Performance in Organizational Identification, Adjustment and Job Performance", Journal of Management Studies, 44 (6), 972-992.

CARROLL, A.B. (2008): "A History of Corporate Social Responsibility: Concepts and Practices". In: Crene, A. (Ed.), The Oxford Handbook of Corporate Social Responsibility, Oxford University Press, Oxford, 19-46.

CARROLL, A.B. \& SHABANA, K.M. (2010): "The Business Case for Corporate Social Responsibility: A Review of Concepts, Research and Practice", International Journal of Management Reviews, 12 (1), 85-105.

CHATTERJEE, S. (2013): "Simple rules for designing business models", California Management Review, 55 (2), 97-124.

CHEAH, E., JAMALI, D., JOHNSON, J.E. \& SUNG, M. (2011): "Drivers of Corporate Social Responsibility Attitudes: The Demography of Socially Responsible Investors", British Journal of Management, 22 (2), 305-323.

CHILD, J. \& GUNTHER, R. (2001). "Organizations unfettered: Organizational form in an information intensive economy", Academy of Management Journal, 44 (6), 1135-1148.

CHIN, M., HAMBRICK, D. \& TREVIÑO, L. (2013): "Political Ideologies of CEOs: The Influence of Executives' Values on Corporate Social Responsibility", Administrative Science Quarterly, 58 (2), 197-232. 
CHRISTENSEN, L., MACKEY, A. \& WHETTEN, D. (2014): "Taking responsibility for corporate social responsibility: The role of leaders in creating, implementing, sustaining, or avoiding socially responsible firm behaviors", Academy of Management Perspectives, 28 (2), 164-178.

CHUN, J., SHIN, Y., CHOI, J. \& KIM, M. (2013): "How Does Corporate Ethics Contribute to Firm Financial Performance?: The Mediating Role of Collective Organizational Commitment and Organizational Citizenship Behavior", Journal of Management, 39 (4), 853-877.

CLEGG, S., KORNBERGER, M. \& RHODES, C. (2007): "Business Ethics as Practice", British Journal of Management, 18 (2), 107-122.

CONTU, A. (2014): "Rationality and Relationality in the Process of Whistleblowing: Recasting Whistleblowing Through Readings of Antigone", Journal of Management Inquiry, 23 (4), 393-406.

CORDING, M., HARRISON, J.S., HOSKISSON, R.E. \& JONSEN, K. (2014): "Walking the talk: A multistakeholder exploration of organizational authenticity, employee productivity, and post-merger performance", Academy of Management Perspectives, 28 (1), 38-56.

CRANE, A., PALAZZO, G., SPENCE, L.J. \& MATTEN, D. (2014): "Contesting the value of "creating shared value", California Management Review, 56 (2), 130-153.

DE CREMER, D., VAN DICK, R., TENBRUNSEL, A., PILLUTLA, M. \& MURNIGHAN, J.K. (2011): "Understanding Ethical Behavior and Decision Making in Management: A Behavioural Business Ethics Approach", British Journal of Management, 22, S1-S4.

DECKOP, J.R. (2006): "The Effects of CEO Pay Structure on Corporate Social Performance", Journal of Management, 32 (3), 329-342.

DELIOS, A. (2010): "How Can Organizations Be Competitive but Dare to Care?", Academy of Management Perspectives, 24 (3), 25-36.

DELMAS, M.A., ETZION, D., NAIRN-BIRCH, N. (2013): "Triangulating environmental performance: What do corporate social responsibility ratings really capture?", Academy of Management Perspectives, 27 (3), 255-267.

DEN HOND, F., REHBEIN, K., DE BAKKER, F. \& LANKVELD, H. (2014): "Playing on two chessboards: Reputation Effects between Corporate Social Responsibility (CSR) and Corporate Political Activity (CPA)", Journal of Management Studies, 51 (5), 790-813.

DEVINNEY, T.M. (2009): "Is the Socially Responsible Corporation a Myth? The Good, the Bad, and the Ugly of Corporate Social Responsibility", Academy of Management Perspectives, 23 (2), 4456.

DOH, J.P. \& GUAY, T.R. (2006): "Corporate Social Responsibility, Public Policy, and NGO Activism in Europe and the United States: An Institutional-Stakeholder Perspective", Journal of Management Studies, 43 (1), 47-73. 
DOH, J.P., HOWTON, S.D., HOWTON, S.W. \& SIEGEL, D.S. (2009): "Does the Market Respond to an Endorsement of Social Responsibility? The Role of Institutions, Information, and Legitimacy", Journal of Management, 36 (6), 1461-1485.

DOWLING, G. \& MORAN, P. (2012): “Corporate Reputations: BUILT IN OR BOLTED ON?”, California Management Review, 54 (2), 25-42.

DU, S., BHATTACHARYA, C.B. \& SEN, S. (2010): "Maximizing Business Returns to Corporate Social Responsibility (CSR): The Role of CSR Communication", International Journal of Management Reviews, 12 (1), 8-19.

ECCLES, R.G., NEWQUIST, S.C. \& SCHATZ, R. (2007): "Reputation and its risks", Harvard Business Review, 85 (2), 104-114.

FLAMMER, C. (2013): "Corporate social responsibility and shareholder reaction: The environmental awareness of investors", Academy of Management Journal, 56 (3), 758-781.

FREEMAN, R. (2006): "The Wal-Mart Effect and Business, Ethics, and Society", Academy of Management Perspectives, 20 (3), 38-40.

FRENKEL, S.J. \& SCOTT, D. (2002): "Compliance, Collaboration, and Codes of Labor Practice: The Adidas connection", California Management Review, 45 (1), $29-49$.

GALBREATH, J. (2010): "Drivers of Corporate Social Responsibility: the Role of Formal Strategic Planning and Firm Culture", British Journal of Management, 21 (2), 511-525.

GEORGE, J.M. (2014): "Compassion and Capitalism: Implications for Organizational Studies", Journal of Management, 40 (1), 5-15.

GODFREY, P. \& MAHONEY, J. (2014). "The Functions of the Executive at 75: An Invitation to Reconsider a Timeless Classic", Journal of Management Inquiry, 23 (4), 360-372.

GOOLD, M. \& CAMPBELL, A. (2002): "Do you have a well-designed organization?", Harvard Business Review, 80 (3), 117-124.

HESS, D., ROGOVSKY, N. \& DUNFEE, T.W. (2002): "The Next Wave of Corporate Community Involvement: CORPORATE SOCIAL INITIATIVES", California Management Review, 44 (2), 110125.

HILLENBRAND, C., MONEY, K. \& GHOBADIAN, A. (2013): "Unpacking the Mechanism by which Corporate Responsibility Impacts Stakeholder Relationships", British Journal of Management, 24 (1), 127-146.

HOFFMAN, A.J. (1999): "Institutional evolution and change: Environmentalism and the U.S. chemical industry", Academy of Management Journal, 42, 351-371.

HOLLENDER, J. (2004): "What Matters Most: Corporate Values and Social Responsibility", California Management Review, 46 (4), 111-119. 
HÖLLERER, M.A. (2013): "From Taken-for-Granted to Explicit Commitment: The Rise of CSR in a Corporatist Country", Journal of Management Studies, 50 (4), 573-606.

HUSTED, B.W. \& DE JESUS SALAZAR, J. (2006): "Taking Friedman Seriously: Maximizing Profits and Social Performance", Journal of Management Studies, 43 (1), 75-91.

JACOBS, D.C. (2004): "A Pragmatist Approach to Integrity in Business Ethics", Journal of Management Inquiry, 13 (3), 215-223.

JANNEY, J. \& GOVE, S. (2011): "Reputation and Corporate Social Responsibility Aberrations, Trends, and Hypocrisy: Reactions to Firm Choices in the Stock Option Backdating Scandal", Journal of Management Studies, 48 (7), 1562-1585.

KAPTEIN, M. (2008): "Developing a Measure of Unethical Behavior in the Workplace: A Stakeholder Perspective", Journal of Management, 34 (5), 978-1008.

KARNANI, A. (2011a): "Doing Well by Doing Good: The Grand Illusion", California Management Review, $53(2), 69-86$.

KARNANI, A. (2011b): "CSR stuck in a logical trap: A response to Pietra Rivoli and Sandra Waddock's 'first they ignore you...': The time-context dynamic and corporate responsibility", California Management Review, 53 (2), 105-111.

KLEYN, N., ABRATT, R., CHIPP, K. \& GOLDMAN, M. (2012): "Building a strong corporate ethical identity: Key findings from suppliers", California Management Review, 54 (3), 61-76.

KOCK, C.J., SANTALÓ, J. \& DIESTRE, L. (2012). "Corporate Governance and the Environment: What Type of Governance Creates Greener Companies?", Journal of Management Studies, 49 (3), 492514.

LAMPIKOSKI, T., WESTERLUND, M., RAJALA, R. \& MÖLLER, K. (2014): "Green innovation games: Value-creation strategies for corporate sustainability", California Management Review, 57 (1), 88-116.

LANGE, D. \& WASHBURN, N.T. (2012): "Understanding Attributions of Corporate Social Irresponsibility", Academy of Management Review, 37 (2), 300-326.

LAPLUME, A.O., SONPAR, K. \& LITZ, R.A. (2008): "Stakeholder Theory: Reviewing a Theory that Moves Us", Journal of Management, 34 (6), 1152-1189.

LEE, M. (2008): "A review of the theories of corporate social responsibility: Its evolutionary path and the road ahead", International Journal of Management Reviews, 10 (1), 53-73.

LÉVY, M., ESKEW, M., BERNOTAT, W.H. \& BARNER, M. (2007): "Who owns the long term? Perspectives from global business leaders", Harvard Business Review, 85 (7-8), 54-60.

LINDGREEN, A. \& SWAEN, V. (2010): "Corporate Social Responsibility", International Journal of Management Reviews, 12 (1), 1-7. 
LLLIA, L., ZYGLIDOPOULOS, S., ROMENTI, S., RODRIGUEZ-CANOVAS, B. \& DEL VALLE BRENA, A. (2013): "Communicating corporate social responsibility to a cynical public", MIT Sloan Management Review, 54 (3), 2-4.

LOCKETT, A., MOON, J. \& VISSER, W. (2006): "Corporate Social Responsibility in Management Research: Focus, Nature, Salience and Sources of Influence*”, Journal of Management Studies, 43 (1), 115-136.

MACKEY, A., MACKEY, T.B. \& BARNEY, J.B. (2007): "Corporate social responsibility and firm performance: Investor preferences and corporate strategies", Academy of Management Review, 32 (3), 817-835.

MAON, F., LINDGREEN, A. \& SWAEN, V. (2010): "Organizational Stages and Cultural Phases: A Critical Review and a Consolidative Model of Corporate Social Responsibility Development", International Journal of Management Reviews, 12 (1), 20-38.

MARCUS, A.A. \& ANDERSON, M.H. (2006): "A General Dynamic Capability: Does it Propagate Business and Social Competencies in the Retail Food Industry?", Journal of Management Studies, 43 (1), 19-46.

MARGOLIS, J.D. \& WALSH, J.P. (2003): "Misery Loves Companies: Rethinking Social Initiatives by Business", Administrative Science Quarterly, 48 (2), 268-305, SAGE Publications.

MARTIN, R.L. (2002). "The virtue matrix. Calculating the return on corporate responsibility". Harvard Business Review, 80 (3), 68-75.

MATTEN, D. \& MOON, J. (2008): "Implicit and Explicit CSR: A Conceptual Framework for a Comparative Understanding of Corporate Social Responsibility", Academy of Management Review, 33 (2), 404-424.

MCLACHLAN, J. \& GARDNER, J. (2004): "A comparison of socially responsible and conventional investors", Journal of Business Ethics, 52, 11-25.

MCWILLIAMS, A. \& SIEGEL, D. (2001): "Corporate social responsibility: A theory of the firm perspective", Academy of Management Review, 26 (1), 117-127.

MCWILLIAMS, A. \& SIEGEL, D. (2011): "Creating and Capturing Value: Strategic Corporate Social Responsibility, Resource-Based Theory, and Sustainable Competitive Advantage", Journal of Management, 37 (5), 1480-1495.

MCWILLIAMS, A., SIEGEL, D. \& WRIGHT, P.M. (2006): "Corporate Social Responsibility: Strategic Implications", Journal of Management Studies, 43 (1), 1-18.

MENA, S. \& WAEGER, D. (2014): "Activism for corporate responsibility: Conceptualizing private regulation opportunity structures", Journal of Management Studies, 51 (7), 1091-1117.

MINOR, D. \& MORGAN, J. (2011): "CSR as Reputation Insurance: Primum Non Nocere", California Management Review, 53 (3), 40-59. 
MINTZBERG, H., SIMONS, R. \& BASU, K. (2002): "Beyond selfishness", MIT Sloan Management Review, 44 (1), 67-74.

MIRVIS, P. (2012): "Employee engagement and CSR: Transactional, relational, and developmental approaches", California Management Review, 54 (4), 93-117.

MUTHURI, J.N., MATTEN, D. \& MOON, J. (2009): "Employee Volunteering and Social Capital: Contributions to Corporate Social Responsibility", British Journal of Management, 20 (1), 75-89.

NONAKA, I. \& TAKEUCHI, H. (2011): "The wise leader”, Harvard Business Review, 89 (5), 58-67.

OIKONOMOU, I., BROOKS, C. \& PAVELIN, S. (2014): "The financial effects of uniform and mixed corporate social performance", Journal of Management Studies, 51 (6), 898-925.

OOSTERHOUT, J.H.V., HEUGENS, P.M.A.R. \& KAPTEIN, M. (2006): "The internal morality of contracting: Advancing the contractualist endeavor in business ethics", Academy of Management Review, 31 (3), 521-539.

ORLITZKY, M. (2013): "Corporate social responsibility, noise, and stock market volatility", Academy of Management Perspectives, 27 (3), 238-254.

PEARCE, J.A. \& DOH, J.P. (2005): "The high impact of collaborative social initiatives", MIT Sloan Management Review, 46 (3), 30-39.

PELOZA, J. \& FALKENBERG, L. (2009): "The role of collaboration in achieving corporate social responsibility objectives", California Management Review, 51 (3), 95-113.

PELOZA, J., LOOCK, M., CERRUTI, J. \& MUYOT, M. (2012): "Sustainability: How Stakeholder Perceptions Differ from Corporate Reality", California Management Review, 55 (1), 74-97.

PENG, M.W. \& PLEGGENKUHLE-MILES, E.G. (2009): "Current debates in global strategy", International Journal of Management Reviews, 11 (1), 51-68.

PORTER, M. \& KRAMER, M. (2002): "The competitive advantage of corporate philanthropy", Harvard Business Review, 80 (12), 57-68.

PORTER, M.E. \& KRAMER, M.R. (2006): "Strategy y society: The link between competitive advantage and corporate social responsibility", Harvard Business Review, 84 (12), 78-92.

RAAR, J. (2002): "Environmental initiatives: towards triple-bottom line reporting", Corporate Communications: An International Journal, 7 (3), 169-183.

RAFFAELLI, R. \& GLYNN, M. (2014): "Turnkey or tailored? relational pluralism, institutional complexity, and the organizational adoption of more or less customized practices", Academy of Management Journal, 57 (2), 541-562.

RIVOLI, P. \& WADDOCK, S. (2011): "First they ignore you...: The time-context dynamic and corporate responsibility", California Management Review, 53 (2), 87-104. 
ROSS, D. (2014): "Taking a chance: A formal model of how firms use risk in strategic interaction with other firms", Academy of Management Review, 39 (2), 202-226.

SALGE, T. \& VERA, A. (2013): "Small Steps that Matter: Incremental Learning, Slack Resources and Organizational Performance", British Journal of Management, 24 (2), 156-173.

SCHERER, A.G. \& PALAZZO, G. (2007): "Toward a political conception of corporate responsibility: Business and society seen from a habermasian perspective", Academy of Management Review, 32 (4), 1096-1120.

SCHERER, A.G. \& PALAZZO, G. (2011): "The New Political Role of Business in a Globalized World: A Review of a New Perspective on CSR and its Implications for the Firm, Governance, and Democracy", Journal of Management Studies, 48 (4), 899-931.

SCHERER, A.G., PALAZZO, G. \& SEIDL, D. (2013): "Managing Legitimacy in Complex and Heterogeneous Environments: Sustainable Development in a Globalized World", Journal of Management Studies, 50 (2), 259-284.

SCHULER, D.A. \& CORDING, M. (2006): "A corporate social performance corporate financial performance behavioral model for consumers", Academy of Management Review, 31 (3), 540-558.

SECCHI, D. (2007): "Utilitarian, managerial and relational theories of corporate social responsibility", International Journal of Management Reviews, 9 (4), 347-373.

SHARRATT, D., BRIGHAM, B.H. \& BRIGHAM, M. (2007): "The Utility of Social Obligations in the UK Energy Industry*", Journal of Management Studies, 44 (8), 1503-1522.

SMITH, N.C. (2003): "Corporate Social Responsibility: Whether or How?", California Management Review, 45 (4), 52-76.

SOULE, E. (2002): "Managerial moral strategies in search of a few good principles", Academy of Management Review, 27 (1), 114-124.

SURROCA, J., TRIBÓ, J.A. \& ZAHRA, S.A. (2013): "Stakeholder pressure on MNEs and the transfer of socially irresponsible practices to subsidiaries", Academy of Management Journal, 56 (2), 549572.

TANG, Z., HULL, C.E. \& ROTHENBERG, S. (2012): "How Corporate Social Responsibility Engagement Strategy Moderates the CSR-Financial Performance Relationship", Journal of Management Studies, 49 (7), 1274-1303.

TREVINO, L.K., WEAVER, G.R. \& REYNOLDS, S.J. (2006): "Behavioral Ethics in Organizations: A Review", Journal of Management, 32 (6), 951-990.

VALLESTER, C., LINDGREEN, A., MAON, F., KLEYN, N., ABRATT, R., CHIPP, K. \& GOLDMAN, M. (2012): "Strategically Leveraging Corporate Social Responsibility: A Corporate Branding Perspective", California Management Review, 54 (3), 34-60. 
VOGEL, D.J. (2005). "Is There a Market for Virtue? The business case for Corporate Social Responsibility", California Management Review, 47 (4), 19-45.

WADDOCK, S. (2000): "The Multiple Bottom Lines of Corporate Citizenship:Social Investing, Reputation, and Responsibility Audits", Business and Society Review, 105 (3), 323-345.

WADDOCK, S. (2008): "Building a New Institutional Infrastructure for Corporate Responsibility", Academy of Management Perspectives, 22 (3), 87-108.

WADDOCK, S. \& GRAVES, S.B. (1997): "The corporate social performance-financial performance link", Strategic Management Journal, 18, 303-319.

WALDMAN, D.A. \& SIEGEL, D. (2008): "Defining the socially responsible leader", The Leadership Quarterly, 19, 117-131.

WALDMAN, D.A., SIEGEL, D.S. \& JAVIDAN, M. (2006): "Components of CEO Transformational Leadership and Corporate Social Responsibility", Journal of Management Studies, 43 (8), 17031725 .

WANG, H. \& CHOI, J. (2013): "A New Look at the Corporate Social-Financial Performance Relationship: The Moderating Roles of Temporal and Interdomain Consistency in Corporate Social Performance", Journal of Management, 39 (2), 416-441.

WARREN, R. \& TWEEDALE, G. (2002): "Business Ethics and Business History: Neglected Dimensions in Management Education", British Journal of Management, 13 (3), 209-219.

WEAVER, G., REYNOLDS, S. \& BROWN, M. (2014): "Moral Intuition: Connecting Current Knowledge to Future Organizational Research and Practice", Journal of Management, 40 (1), 100-129.

WELSH, D. \& ORDÓÑEZ, L. (2014): "Conscience without cognition: The effects of subconscious priming on ethical behavior", Academy of Management Journal, 57 (3), 723-742.

WINDSOR, D. (2006): "Corporate Social Responsibility: Three Key Approaches", Journal of Management Studies, 43 (1), 93-114.

WOOD, D. (1991). "Corporate social performance revisited", Academy of Management Review, 16, 691-718.

WOOD, D. (2010): "Measuring Corporate Social Performance: A Review", International Journal of Management Reviews, 12 (1), 50-84.

ZADEK, S. (2004): "The path to corporate responsibility", Harvard Business Review, 82 (12), 125-132. 
